C-A/AP/\#157 June 2004

\title{
Feasibility Study of a 1.5-GeV Proton FFAG in the AGS Tunnel
}

\author{
Alessandro G. Ruggiero
}

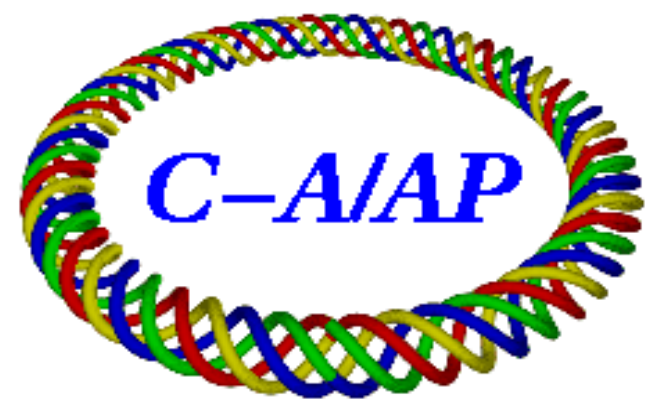

Collider-Accelerator Department Brookhaven National Laboratory Upton, NY 11973 


\title{
Feasibility Study of a 1.5-GeV Proton FFAG in the AGS Tunnel"
}

\author{
Alessandro G. Ruggiero \\ Brookhaven National Laboratory \\ June 16, 2004
}

\begin{abstract}
A 1.5-GeV Fixed-Field Alternating-Gradient (FFAG) proton Accelerator is being studied as a new injector to the Alternating-Gradient Synchrotron (AGS) of Brookhaven National Laboratory (BNL). The major benefit is that it would considerably shorten the overall AGS acceleration cycle, and, consequently, may yield to an improvement of beam stability, intensity and size. The AGS-FFAG, as we shall call the new injector, will also facilitate the proposed upgrade of the AGS facility toward a 1-MW average proton beam power at the top energy of $28 \mathrm{GeV}$. This paper describes the FFAG design for acceleration of protons from $400 \mathrm{MeV}$ to $1.5 \mathrm{GeV}$, with the same circumference of the AGS, and entirely housed in the AGS tunnel. The lattice is a periodic sequence of FDF triplets of combined-function magnets. An adjusted magnet field profile has been calculated to compensate the variation of the main lattice functions with momentum. At injection, a 1.0-ms long beam pulse of negative-ions $\left(\mathrm{H}^{-}\right)$is stacked using the chargeexchange method. Acceleration of one pulse with $1.0 \times 10^{14}$ protons takes about $7.0 \mathrm{msec}$. The beam is transferred in one single turn from the FFAG to the AGS.
\end{abstract}

\section{Introduction}

The concept of the Fixed-Field Alternating-Gradient (FFAG) accelerator was introduced in the middle of last century [1]. Several electron accelerators adopting the concept of fixed field were demonstrated [2]. Acceleration of protons was proposed at different times [3], but none of these projects materialized. Only recently acceleration of protons in relatively low-energy $(10-150 \mathrm{MeV})$ FFAG accelerators was proven at KEK in Japan [4]. There is recently a revival of interest in this type of technology especially for the generation of proton beam power in the MW range, and energy of about one or few $\mathrm{GeV}$. This technology, if successfully demonstrated, would represent a considerable shortcut at a more modest cost and technical investment than the Super-Conducting Linacs that have in the meantime been proposed for the same scope [5]. Our interest in the FFAG proton accelerator is that it can be used as an injector to the AGS to facilitate the mode of operation, to reduce beam losses, to increase the AGS repetition rate, and to achieve a higher proton beam power. But for a reasonable design covering a large momentum range, that yet avoids magnets with too large aperture and excessive chromatic effects, we had to rely on a method that makes use of an adjusted magnet field profile.

\section{The BNL-AGS Facility}

The present BNL-AGS accelerator complex is shown schematically in Figure 1. It is made of four different accelerators: (i) the 200-MeV room-temperature Drift-Tube Linac

\footnotetext{
* Work performed under the auspices of the US Department of Energy
} 
(DTL) operating at $201.25 \mathrm{MHz}$; (ii) the Heavy-Ion Tandems that provide the intense, short beam pulses of Heavy Ions; (iii) the AGS Booster for the acceleration of protons to $1.5 \mathrm{GeV}$, as well of heavier ions to other intermediate energies; (iv) the AlternatingGradient Synchrotron (AGS) that accelerates all type of particles to energy, for instance, of $28 \mathrm{GeV}$ in the case of protons, and $12 \mathrm{GeV} / \mathrm{u}$ for ions of $\mathrm{Au}$.

The AGS-Booster plays a central role for the collection and preparation of all types of particle beams before they are transferred to the AGS for the final acceleration proper. Yet the Booster is a relatively slow-cycling accelerator, and several Booster cycles are required for the complete filling of the AGS. For instance, a typical mode of operation for protons requires that negative ions $\left(\mathrm{H}^{-}\right)$are injected into the Booster at $200 \mathrm{MeV}$ and accumulated by charge-exchange method. The emerging protons are then accelerated to

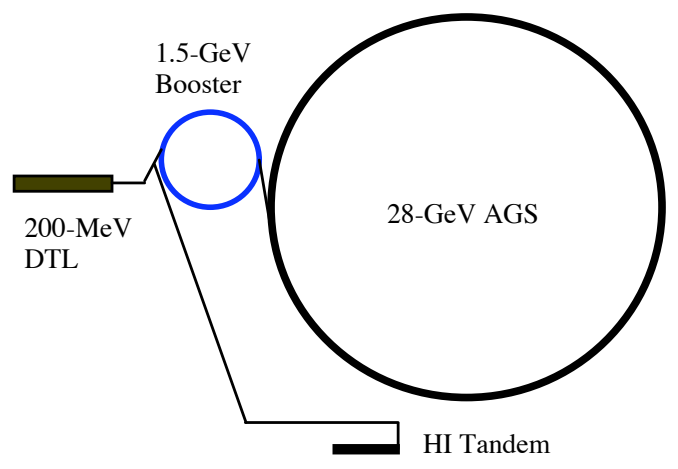

Figure 1. Present AGS Accelerator Complex 1.5 GeV and transferred to the AGS that is initially set in a holding mode. The AGS circumference is four times that of the Booster, so that a complete fill of the AGS requires also four Booster beam pulses. Once the AGS is completely filled, the whole beam is then accelerated to the top energy of $28 \mathrm{GeV}$. Presently, the repetition rate of the Booster is $7.5 \mathrm{~Hz}$, and that of the AGS is $0.5 \mathrm{~Hz}$. That requires a filling time of the AGS of about 0.5 second, followed by about one second for acceleration to the top energy, and another second for resetting the AGS field cycle. At best, the overall cycle period is 2.5 seconds. With a typical intensity of $7 \times 10^{13}$ protons per cycle accelerated to $28 \mathrm{GeV}$, that yields an average proton beam power of about $125 \mathrm{~kW}$. It is to be noticed that a filling time of 0.5 second is a long period of time where several effects on the stored beam may occur, with consequent losses and size deterioration. One would expect a great improvement on the beam quality and performance if the filling time can be shortened so that particles do not have to wait too long before they are accelerated.

\section{The AGS Upgrade Program}

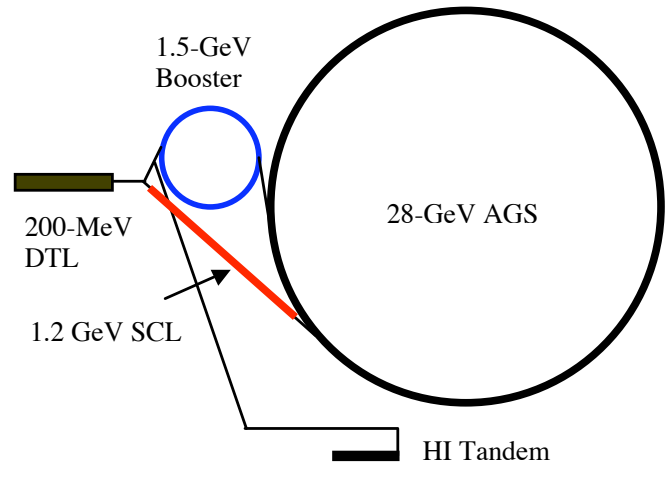

Figure 2. AGS Upgrade with $1.2 \mathrm{GeV}$ SCL
It has been recently proposed [6] to upgrade the AGS facility for an average proton beam power of at least one MW. This can be accomplished in two steps: (1) the replacement of the main magnet AGS power supply with one operating at $2.5 \mathrm{~Hz}$, and (2) a SuperConducting Linac (SCL) for direct acceleration of protons (actually $\mathrm{H}^{-}$) from the $200-\mathrm{MeV}$ DTL to $1.2 \mathrm{GeV}$, followed by immediate transfer into the AGS, as shown in Figure 2. This mode of operation completely bypasses the Booster, and eliminates the $0.5 \mathrm{~s}$ long 
injection period. It is then reasonable to expect a significant improvement on the beam performance, and a 30 percent increase in overall intensity in the AGS to about $10^{14}$ protons per pulse. The shorter is the period of time the beam spends circulating in the AGS, the more stable it is, and it can carry more intensity. A feasibility study of the 1.2GeV SCL has already been done [7]. Such a device is relatively expensive, and is also a technology that still needs to be completely demonstrated $[8,9]$. On the other end, a 1.5$\mathrm{GeV}$ FFAG accelerator represents a valid alternative to the $1.2-\mathrm{GeV}$ SCL, fulfilling the same power requirements. Presently, to ease the design of the SCL proper, it has also been planned to raise the energy of the DTL from 200 to $400 \mathrm{MeV}$. We shall assume such upgrade in energy of the DTL also with the study of the FFAG injector.

\section{Advantages of the FFAG accelerator}

The bending and focusing magnetic fields are kept constant during acceleration. The main power supply is thus continuous. The magnets are not ramped, either way, and the resulting cycle can be made considerably shorter. Assuming the FFAG ring has the same circumference of the AGS and is installed above the AGS magnet in the same tunnel, an energy gain of $0.5 \mathrm{MeV} /$ turn requires a total acceleration cycle of about $7.0 \mathrm{msec}$. Only a single acceleration cycle in the FFAG is required to get a proton beam of the desired intensity that can then be transferred in a single turn to the AGS, and immediately accelerated to the top energy of $28 \mathrm{GeV}$ at the repetition rate of $2.5 \mathrm{~Hz}$. Because the beam spends at most a few thousand revolutions circulating in the FFAG, magnet imperfections and other errors may not have the time to impact significantly on the beam dynamics, and the whole process can be easily computer simulated.

The proton beam is provided by the DTL upgraded to $400 \mathrm{MeV}$, and the overall layout is shown in Figure 3. The full intensity of $1.0 \times 10^{14}$ protons per FFAG pulse can

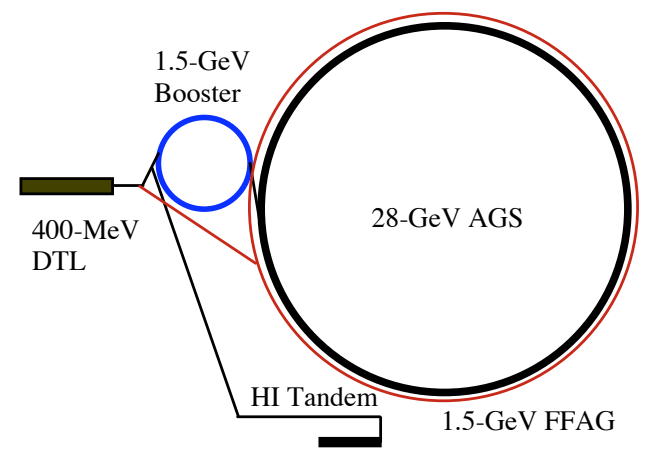

Figure 3. AGS Upgrade with 1.5-GeV FFAG In the AGS Tunnel be attained with multi-turn injection at 400 $\mathrm{MeV}$ by charge exchange. To get the required number of protons per FFAG pulse, one needs a Linac pulse $1.0 \mathrm{~ms}$ long at the rate of 2.5 cycles/second. Since at injection into the FFAG the revolution period is $3.78 \mu \mathrm{s}$, a total of about 260 turns need to be injected. The lattice is compact with stronger focusing yielding smaller lattice functions. At the same time the magnets will have adequate physical aperture to safely confine the beam and to avoid activation by beam losses.

\section{FFAG Accelerator Issues}

The same RF cavity system has to provide acceleration simultaneously to different parts of the beam that cross the cavity with different momentum value and at different radial displacement. Thus the width of the RF cavity has to be large enough to accommodate the whole momentum range of the beam during acceleration. It would be 
desirable that the beam crosses the cavity (or cavities) always in phase. This requires that the motion is isochronous, that is any particle takes the same time to go around one revolution. The revolution period in reality varies for two reasons: (a) the particle velocity varies, and (b) the path length varies, from one orbit to the next. For protons the variation of the path length has a negligible contribution to the transit time, whereas the variation of the beam velocity is more important. It is then more difficult for protons to find ways to fulfill synchronism. This could be achieved only with proper shaping of the magnets longitudinal length. As an alternative one simply requires that the RF frequency is modulated turn after turn, that is versus momentum, and correspondingly versus the radial position of the beam.

The most important issue is the search for an ideal lattice that accommodates such a larger momentum aperture that in our case extends over $\pm 40 \%$. Without entering too much in the detailed discussion of scaling versus non-scaling lattices, it will suffice to say here that it is certainly highly desirable to find a magnet arrangement where the lattice functions, for instance the betatron tunes, do not change too much over the required momentum range. It is almost impossible to find a scaling lattice for a $1.5 \mathrm{GeV}$ proton FFAG, especially if one wants to limit the strength of the bending and focusing fields, that otherwise may require magnets with too large radial aperture. We adopted a non-scaling lattice based on the FDF triplet that requires only modest fields. But we made use of an invention that minimize the variation of the lattice functions with momentum. Essentially, this is achieved with a proper choice of the radial field profile that cancels, at least partially, the variation of the restoring forces with momentum.

\section{The Lattice of the AGS-FFAG}

The FFAG accelerator has a circumference of $807.091 \mathrm{~m}$, equal to that of the AGS, and is made of $\mathrm{N}_{\mathrm{P}}=136$ identical periods, each $5.935 \mathrm{~m}$ long. The period, shown in Fig. 4 , is made of three combined-function sector magnets in the symmetric FDF arrangement. The trajectory shown is that of the reference momentum value $\mathrm{p}_{0}$ taken at injection as shown in Table 1. The circumference of the ring quoted above corresponds to the reference (or injection) trajectory. We shall use the parameter $\delta$ to define the momentum of any particle $\mathrm{p}=\mathrm{p}_{0}(1+\delta)$. At injection $\delta=0$.

Table 1. Proton Beam Kinematic Parameters

Injection $\quad$ Extraction

\begin{tabular}{lll} 
Kinetic Energy, MeV & \multicolumn{1}{c}{400} & \multicolumn{1}{c}{1,500} \\
$\beta$ & 0.713056 & 0.922996 \\
$\gamma$ & 1.42632 & 2.59868 \\
Momentum, MeV/c & 954.263 & $2,250.51$ \\
Magnetic Rigidity, kG-m & 31.8308 & 75.0691 \\
Momentum Deviation, $\delta$ & 0 & 1.36
\end{tabular}




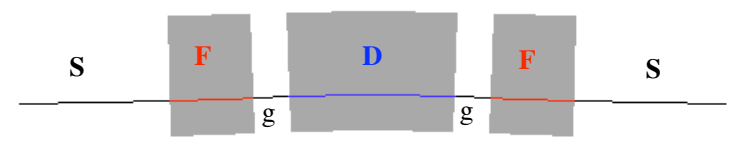

Figure 4. The AGS-FFAG Period (FDF) and the Injection Trajectory.

To notice that the entrance and exit faces of the D-sector magnet are geometrically parallel to the neighboring ones of the two F-sector magnets. Thus, the entrance and exit angles the injection trajectory makes with each magnet are identically equal to zero. This is not true for an off-momentum trajectory. The lattice parameters are shown in Table 2. The $\mathrm{F}$ and $\mathrm{D}$ magnets are separated by a drift $\mathrm{g}=0.3 \mathrm{~m}$; and a long insertion $\mathrm{S}=2.534 \mathrm{~m}$ separates the FDF periods from each other.

Table 2. Magnet Parameters for the Injection Orbit

\begin{tabular}{lll} 
Magnet Type & \multicolumn{1}{c}{ F } & \multicolumn{1}{c}{ D } \\
Arc Length, $\mathrm{m}$ & 0.70 & 0.70 \\
Bending Field B, kG & -0.78409 & 1.8345 \\
Gradient G, kG/m & 26.5817 & -23.2956 \\
Bending Radius $\rho, \mathrm{m}$ & -40.5958 & 17.3512 \\
Bending Angle, mrad & -17.2432 & 80.6862
\end{tabular}

The values shown in Table 2 correspond to the injection trajectory. Whereas the central D-sector magnet bends inward, the two F-sector magnets bend outward; but parameters are adjusted so that the overall bending angle per period is $2 \pi / \mathrm{N}_{\mathrm{p}}$. Thus, since we are assuming circulation of protons (with positive electric charge) in the clockwise direction, the bending field $B$ and the curvature $h=1 / \rho$ in the D-sector magnet are positive, and negative in the F-sector magnets. The lattice functions along the length of one period on the injection trajectory are displayed in Figure 5. The amplitude lattice functions $\beta_{\mathrm{H}}$ and $\beta_{\mathrm{V}}$ have smaller values than those corresponding to the present Booster lattice. The dispersion $\eta$ is smaller in the middle of the D-sector magnet, and larger in the long-drift S. The lattice parameters are summarized in Table 3. The phase advance per period is around $100^{\circ}$ in both planes, and the betatron tunes differ from each other by two units to avoid the effect of a coupling resonance. Because of the reverse bending the transition energy is pure imaginary.

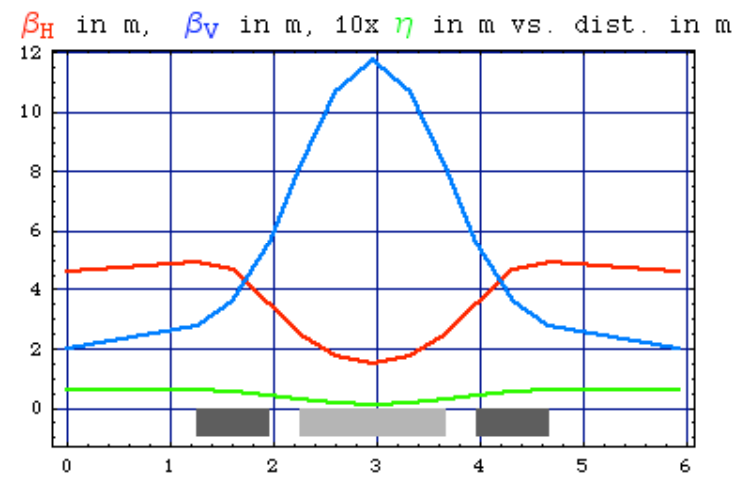

Figure 5. The AGS-FFAG Lattice Functions for the Injection Orbit $(\delta=0)$ 
Table 3. The AGS-FFAG Parameters for the Reference Trajectory

\author{
Circumference \\ Number of Periods \\ Period Length \\ Period Structure \\ Short Drift, g \\ Long Drift, $S$ (total) \\ $\beta_{\mathrm{H}} \max ($ in $\mathrm{S}$ ) \\ $\beta_{\mathrm{v}} \max ($ in $\mathrm{D})$ \\ $\eta \max ($ in $\mathrm{S}$ ) \\ Phase Advance / Period, H/V \\ Betatron Tunes, $\mathrm{H} / \mathrm{V}$ \\ Natural Chromaticity, H/V \\ Transition Energy, $\gamma_{\mathrm{T}}$
}

$$
\begin{aligned}
& 807.091 \mathrm{~m} \\
& 136 \\
& 5.93449 \mathrm{~m} \\
& \mathrm{~S} F \mathrm{~g} \mathrm{D} g \mathrm{~F} \\
& 0.30 \mathrm{~m} \\
& 2.53450 \mathrm{~m} \\
& 4.5733 \mathrm{~m} \\
& 11.7902 \mathrm{~m} \\
& 0.060 \mathrm{~m} \\
& 105.234^{\circ} / 99.9345^{\circ} \\
& 39.755 / 37.755 \\
& -0.9263 /-1.8052 \\
& 105.482 \mathrm{i}
\end{aligned}
$$

\section{Adjusted Field Profile}

At the beginning of our study we found very difficult to find a lattice solution stable over the required momentum aperture with a linear field profile. We were eventually able to find such a stable solution; but, since we were adopting a non-scaling lattice, we were frustrated by the too large variations of the lattice functions from injection to extraction. We began then looking for ways to optimize the field profile to yield the smaller variation of the lattice functions as possible.

The linear equations of motion for a particle with electric charge $q$ and momentum $p$ moving in a constant magnetic field with strength $B$ and gradient $G$ are

$$
\begin{array}{llll}
\mathrm{x}^{\prime \prime}+(\mathrm{hB}+\mathrm{G}) \mathrm{qx} / \mathrm{cp} & = & \mathrm{h}\left(1-\mathrm{p}_{0} / \mathrm{p}\right) \\
\mathrm{y}^{\prime \prime} & =\mathrm{qGy/cp} & 0
\end{array}
$$

The dependence of the particle momentum $\mathrm{p}=\mathrm{p}_{0}(1+\delta)$ does not enter in the curvature function $\mathrm{h}(\mathrm{s})$ that has a pure geometric definition, but enters as the denominator of the field components. This has the effect to introduce large chromatic effects that need to be compensated. First of all we found convenient to tune the accelerator ring at the lowest momentum value corresponding to injection $(\delta=0)$ and require the motion to be stable there. We then offset the momentum dependence allowing the gradient $\mathrm{G}$ to vary radially (x). Since ultimately $x=x(\delta)$ and $G=G(x)$, we can set the relation between $G$ and $\delta$, for both sector magnets, as follows

$\mathrm{G}(\mathrm{x})=\mathrm{G}_{\mathrm{F}, \mathrm{D}}[1+\delta(\mathrm{x})]$

where $\mathrm{G}_{\mathrm{F}, \mathrm{D}}$ are the values shown in Table 2 that correspond to the injection trajectory. We have left the momentum dependence on the curvature term at the right hand side of Eq. (1) unchanged. That is the source of dispersion. Thus our approach is clear [10]: with relation (3) inserted in the equations of motion, the momentum dependence is, at least 
partially, offset. We have developed a SYNCH-like code (using MATHEMATICA [11]) where the symbolism represented by Eq. (3) is included. With the use of the powerful symbolic language of MATHEMATICA, it is possible to invert relationships and to derive $\delta$ as a function of displacement $\mathrm{x}$ and the actual field profile versus radial aperture. The results are shown in Figure 6. The bending field is given in kGauss versus the radial location $\mathrm{x}$ with $\mathrm{x}=0$ corresponding to the location of the beam at $400 \mathrm{MeV}$. There are actually five profiles corresponding, in the case of the F-sector magnet, to five different
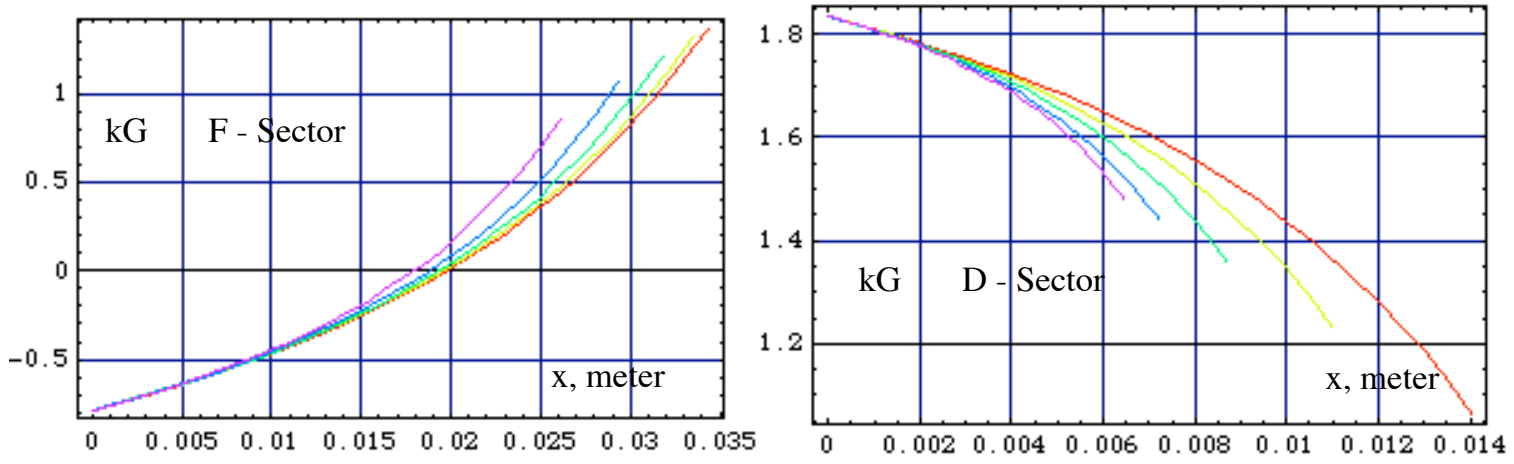

Figure 6. Field Profiles vs. Radial (x) Position

equally spaced locations along the length of the magnet, and, in the case of the D-sector magnet, also to five equally spaced locations but only along half of the length of the magnet since the lattice of a period has a mirror symmetry. Thus the field profile not only is nonlinear but also varies along the length of the magnet. The F-sector magnet is essentially a quadrupole with a field range between -0.8 and +0.8 kGauss centered around $\mathrm{x}=1.85 \mathrm{~cm}$, on top of which nonlinearities are added extending the field to 1.4 kGauss up to about $x=3.4 \mathrm{~cm}$ that corresponds to the location of the beam at $1.5 \mathrm{GeV}$. The D-sector magnet has the typical profile of a combined function magnet (a' la AGS) extending over a narrower radial excursion between $\mathrm{x}=0$ and $1.4 \mathrm{~cm}$.

\section{Momentum Dependence of the AGS-FFAG Lattice}

The fixed closed orbits $x_{P}$ for momentum values in the range $\delta=0-1.36$ are plotted in Figure 7 versus the longitudinal coordinate $s$ across half of a symmetric period. The radial extension of the momentum envelope is reasonably narrow: $3.5 \mathrm{~cm}$ in the middle of the long-straight $\mathrm{S}$, and less than $1 \mathrm{~cm}$ in the center of the D-sector magnet. Figure 8

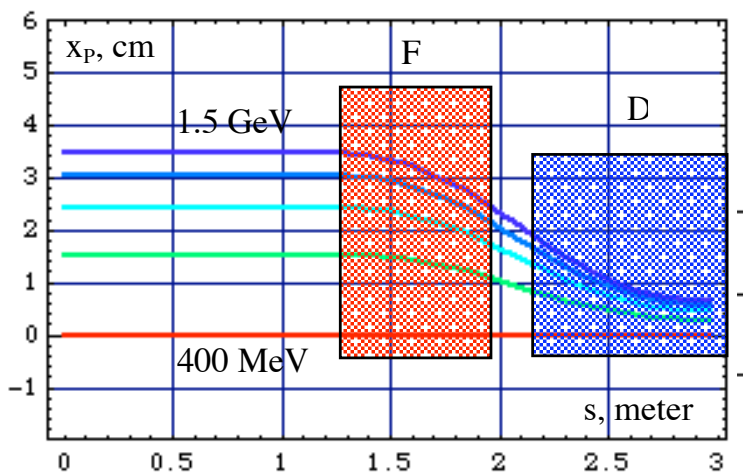

Figure 7. Closed Orbit vs. Path Length

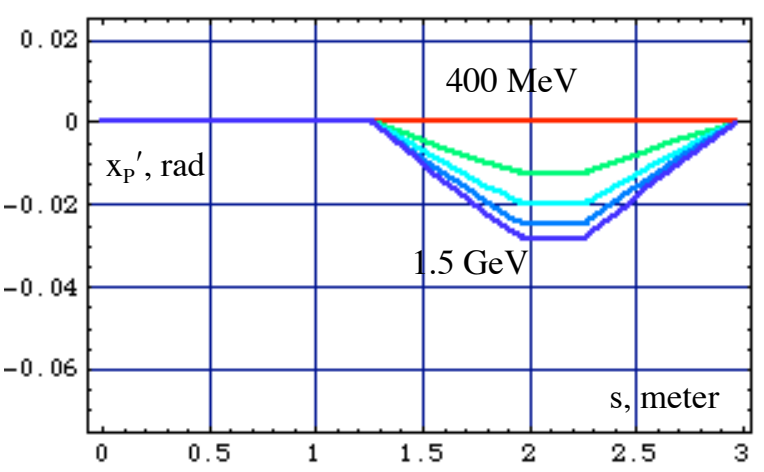

Figure 8. Trajectory Angle $\mathrm{x}_{\mathrm{P}}{ }^{\prime}$ for $\delta=0-1.36$ 
gives the derivative $\mathrm{x}_{\mathrm{P}}{ }^{\prime}$ of the fixed closed orbit radial location $\mathrm{x}_{\mathrm{P}}$ versus the curvilinear coordinate $\mathrm{s}$. The derivative $\mathrm{x}_{\mathrm{p}}{ }^{\prime}$, estimated at the proper locations, gives in turn the entrance and exit angles the off-momentum trajectories make with each of the sector magnets. These angles can be as large as $30 \mathrm{mrad}$. They have a focusing/defocusing effect, and their contribution to the lattice functions has been properly included in our calculation. Finally the fractional parts of the betatron tunes $v_{\mathrm{H}}$ and $v_{\mathrm{V}}$ are plotted in Figure 9 versus the momentum deviation $\delta$. During the entire acceleration cycle each tune does not vary by more than 0.1 , and the variation is caused by the residual focusing effect of the entrance and exit angles of the trajectories discussed above.

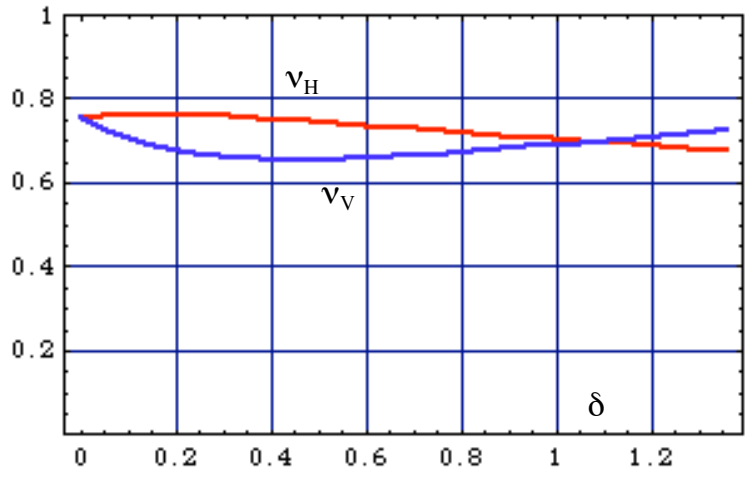

Figure 9. Fractional part of $v_{\mathrm{H}}$ and $v_{\mathrm{V}}$ versus $\delta$

\section{Magnet Considerations}

The field profile in the two sector magnets is shown in Fig. 6. The magnets are eventually made of a sufficiently large number of sectors of constant transverse cross-section. To get the right profile, each sector has an assigned gap and width, the current in each of the four coils can have an asymmetric distribution, and the axial and mid-plane symmetry can be intentionally broken. Programs like

TOSCA or OPERA can eventually be used to design magnets with the proper iron shape to create the required profile. Moreover, because the magnetic field is constant, there are no eddy-currents and the vacuum chamber can be made of solid metallic material of any thickness. The field is constant in time and the magnet iron can be made of solid blocks without lamination. It will be curved to follow closely the curvilinear trajectory. The vacuum chamber is curved and has a constant elliptical cross-section $20 \mathrm{~cm}(\mathrm{H}) \times 10 \mathrm{~cm}$ (V) as shown in Figures 10 all along the length of the magnets. In the long drift $\mathrm{S}$ the vacuum chamber, shown in Figure 11, is straight and has a circular cross-section of 10 $\mathrm{cm}$ diameter. Figures 10 and 11 show also the dimension and location of the beam during the acceleration cycle. Sufficient margin has been allowed in space between the beam boundary and the vacuum chamber to avoid beam losses.

F-sector magnet

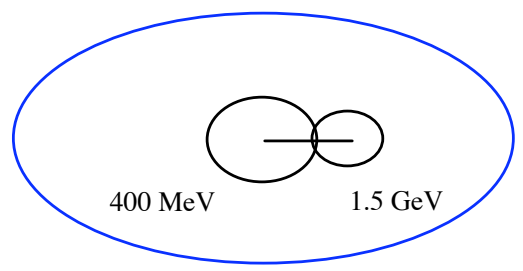

D-sector magnet

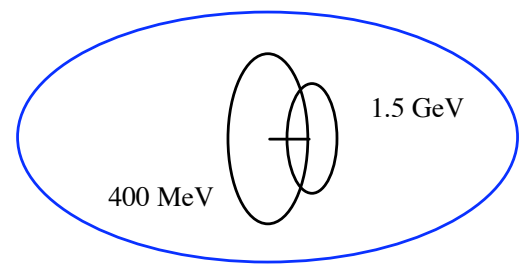

Figure 10. Maximum Beam Dimensions during Acceleration within an Elliptical $20 \mathrm{~cm}$ x $10 \mathrm{~cm}$ Vacuum Chamber 


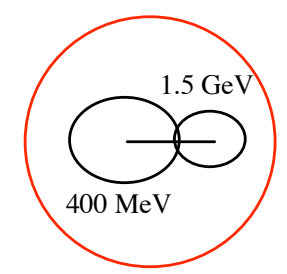

Figure 11. Maximum Beam Dimensions during Acceleration in the Long Straight within a

Circular 10-cm Diameter Vacuum Chamber

\section{Injection of Protons into the AGS-FFAG}

About 260 turns of negative-ions $\mathrm{H}^{-}$are to be injected from the 400-MeV Drift Tube Linac into the AGS-FFAG. The beam will come from an elevation below the FFAG magnets, and then deflected upward on the FFAG mid-plane with two vertical dogleg magnets. Quadrupoles are also inserted along the injection transport line for matching of the lattice functions to values corresponding to injection into the FFAG ring. Prior to the start of injection, a horizontal orbit bump of the injection orbit displaced by $40 \mathrm{~mm}$ is created. The transfer-injection line is shown in Figure 12 where the horizontal dimensions are not in scale. The bump spans over two periods, and is created by two bump magnets B1 and B2 separated ideally by a phase advance of $180^{\circ}$. At the center of the bump, at about the middle of the 2.5-m long drift space (S), there are two horizontal dogleg magnets $\mathrm{C} 1$ and $\mathrm{C} 2$ separated enough for the insertion of a Carbon foil that, traversed by the beam, will cause stripping. The beam rms spot size on the foil is $2.2 \mathrm{~mm}$ $(\mathrm{H}) \times 1.4 \mathrm{~mm}(\mathrm{~V})$. The beam is then captured on the injection orbit by the chargeexchange process. Multi-turn injection last about $1.0 \mathrm{~ms}$. At the end of the process the beam should extend over the length of one turn, with a gap of about $300 \mathrm{~ns}$, and then accelerated.

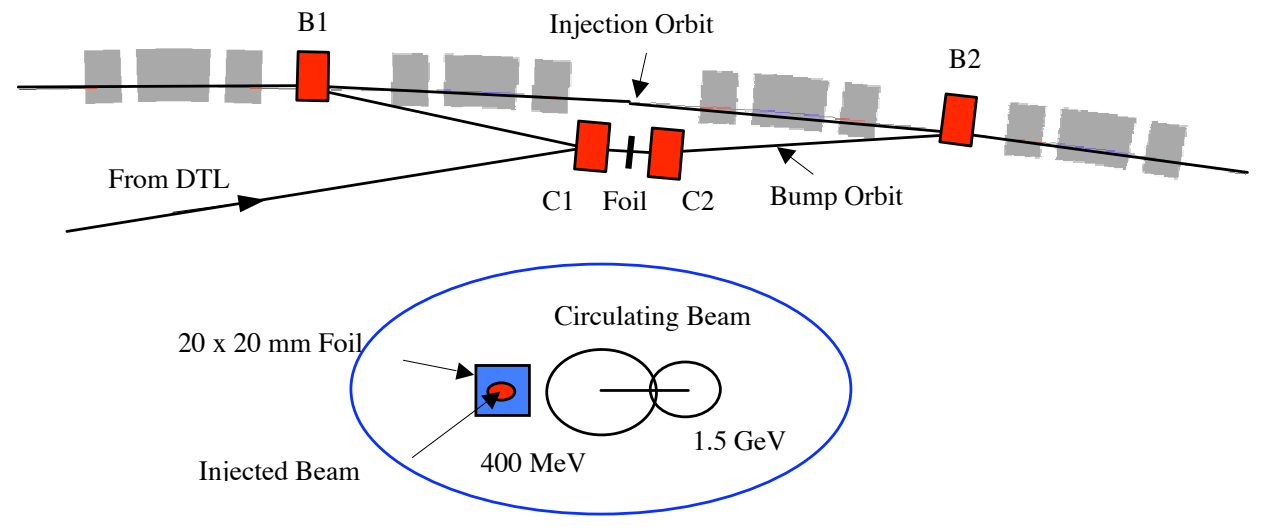

Figure 12. Injection Transfer Line and Beam Cross-Section at the Foil

During injection the accelerating RF is on, set to zero phase angle and constant voltage. The harmonic number is $h=24$. To avoid unnecessary beam losses, the beam is pre-chopped at the beginning of the DTL for the duration of about $50 \%$ of the revolution 
period. The beam from the Linac is bunched at $201.25 \mathrm{MHz}$. Each Linac bunch has a narrow length and an energy spread of about $2.0 \mathrm{MeV}$ (full). The beam dilution during accumulation is controlled by a combination of betatron and momentum painting. The chopping frequency equals the RF frequency at injection. Table 4 gives the summary of the beam parameters at injection.

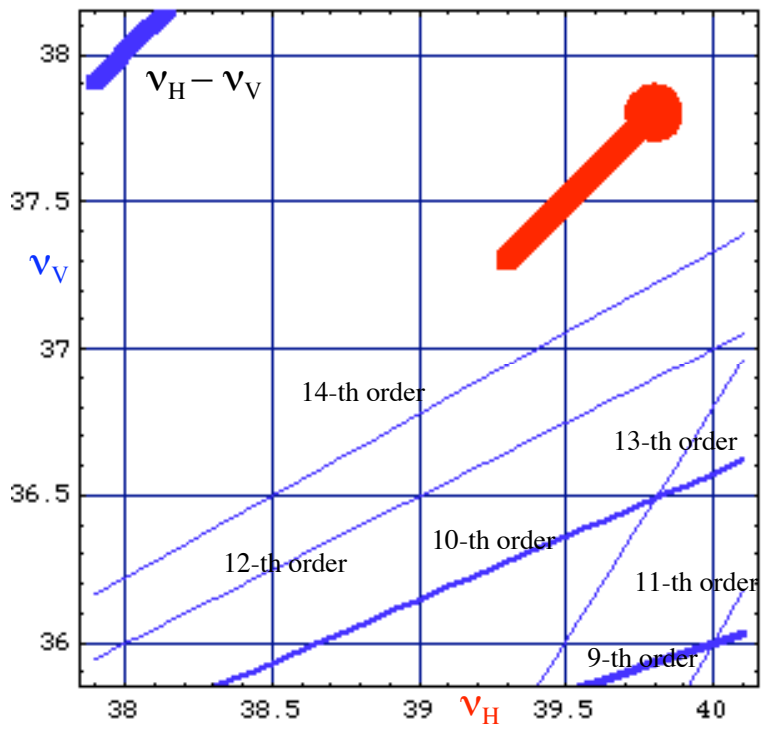

Figure 13. Tune Diagram

At the end of the multi-turn injection, the beam emittance has blown-up to a full value of about $100 \pi \mathrm{mm}$-mrad (normalized and none, since $\beta \gamma \sim 1$ ) corresponding to a space-charge tuneshift of 0.50 , assuming a bunching factor (ratio of beam peak current to average current) of 3 . The maximum tunedepression is reached at the end of multiturn injection, and as soon the beam is accelerated it will decreases again more or less as fast as $\beta \gamma$. The unperturbed tunes have been chosen to avoid the crossing of any major systematic resonance. The tune diagram is shown in Figure 13. It shows all systematic resonances below the $15^{\text {th }}$ order in the square region of two units per side at and below the working point. The nearest lowest order systematic resonance in proximity of the working point (red circle) and of the maximum interval of space-charge tune-shift is a $14^{\text {th }}$ order resonance. We do not expect thus any problem with the structure of the proposed lattice. It remains to investigate the effects of random errors due to magnet imperfections and of the nonlinearities built in the magnets themselves for the attainment of the adjusted field profile. But our concern is greatly reduced because of the large periodicity of the ring.

Table 4. Beam Parameters at Injection into the FFAG

Linac Peak Current

Revolution Period

No. of Protons / FFAG pulse

Chopping Ratio

Chopping Frequency

Single Pulse Length

No. of Turns Injected / pulse

Linac/FFAG repet. rate

Linac Duty Cycle

Linac Beam Emittance, rms norm.

Final Beam Emittance, full norm.

Bunching Factor

Space-Charge Tune-Shift
$35 \mathrm{~mA}$

$3.78 \mu \mathrm{s}$

$1.0 \times 10^{14}$

0.50

$6.357 \mathrm{MHz}$

$0.96 \mathrm{~ms}$

255

$2.5 \mathrm{~Hz}$

$0.24 \%$

$1 \pi \mathrm{mm}-\mathrm{mrad}$

$100 \pi \mathrm{mm}-\mathrm{mrad}$

3

0.50 


\section{Acceleration in the AGS-FFAG}

We opted for the frequency modulation as the beam is accelerated, preserving the constancy of the harmonic number and of the RF voltage. We assumed a constant energy gain of $0.5 \mathrm{MeV} /$ turn and the harmonic number $\mathrm{h}=24$. To create a beam gap of $300 \mathrm{nsec}$ for the extraction kicker, two consecutive out of the $24 \mathrm{RF}$ buckets are void of any beam. Acceleration from $400 \mathrm{MeV}$ to $1.5 \mathrm{GeV}$ takes 2,200 revolutions and lasts $7.0 \mathrm{~ms}$. The required RF swing is shown in Figure 14 and ranges between 6.357 and $8.228 \mathrm{MHz}$. The circulating beam current varies between 4.2 and $5.5 \mathrm{Amp}$, and the beam power has a corresponding range between 2.1 and $2.8 \mathrm{MW}$. The total required RF voltage is about 0.8 MV that can be obtained from 20 single-gap cavities each one meter long, with a peak voltage of $40 \mathrm{kV}$. The cavities are located in pair in the $2.5-\mathrm{m}$ long drifts, and have an internal diameter of $10 \mathrm{~cm}$ to accommodate the beam as shown in Figure 11. Assuming a total peak power requirement of $5 \mathrm{MW}$, each cavity will be driven by a $250 \mathrm{~kW}$ power amplifier. The total cycle period, including $1.0 \mathrm{~ms}$ for multi-turn injection, is $8.0 \mathrm{~ms}$. At the repetition rate of $2.5 \mathrm{~Hz}$, the RF duty cycle is $2.0 \%$. It is also possible to accelerate the beam at a lower rate by lengthening the pulse and reducing the amount of peak power. Conversely, it is also possible to increase the number of cavities in a second stage for a higher acceleration rate. Tables 5 and 6 give a summary of the RF acceleration parameters and of the RF system. At the end of acceleration, just prior to extraction, the RF voltage is lowered so that beam bunching factor of 4 is achieved, that, assuming no dilution of beam emittance at the transfer between FFAG and AGS, will yield a space-charge tune-shift of 0.16 and an actual full emittance of $42 \pi \mathrm{mm}$-mrad. In the locations of large vertical beta-values this gives a full beam height of $60 \mathrm{~mm}$ small enough to be contained in the AGS vacuum chamber. Otherwise, as a good feature of the FFAG, the acceleration range in the FFAG could be extended, for instance to $2 \mathrm{GeV}$ for an optimal matching of beam size and AGS vacuum chamber.

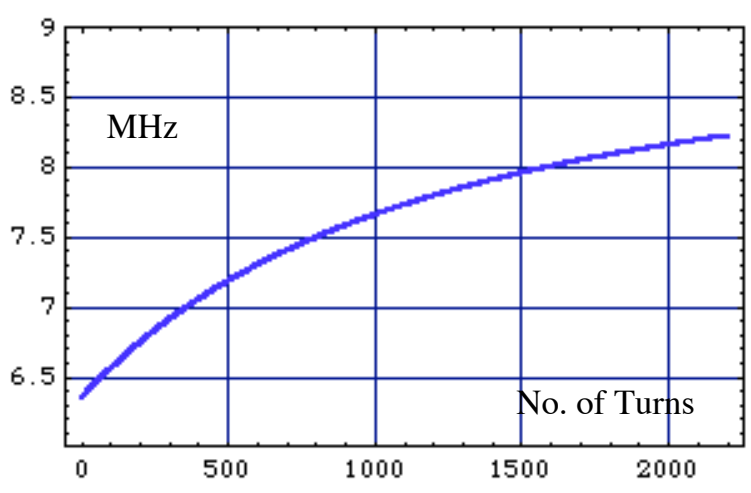

Figure 14. RF Frequency Modulation during Acceleration in the FFAG

Table 5. Parameters of Acceleration in the AGS-FFAG

Circumference

Harmonic Number, $h$

Energy Gain

Transition Energy, $\gamma_{\mathrm{T}}$

Peak RF Voltage

Number of full Buckets

Total Number of Protons

Protons / Bunch

Injection Period

Acceleration Period

Total Cycle Period
$807.091 \mathrm{~m}$

24

$0.5 \mathrm{MeV} /$ turn

$105.5 \mathrm{i}$

$0.8 \mathrm{MVolt}$

22 out of 24

$1.0 \times 10^{14}$

$4.6 \times 10^{12}$

$1.0 \mathrm{~ms}$

$7.0 \mathrm{~ms}$

$8.0 \mathrm{~ms}$ 


$\begin{array}{lll}\text { No. of RF Cavities } & 20 & \\ \text { No. of Gaps per Cavity } & 1 & \\ \text { Cavity Length } & 1.0 \mathrm{~m} & \\ \text { Internal Diameter } & 10 \mathrm{~cm} & \\ \text { Peak Voltage / Cavity } & 40 \mathrm{kVolt} & \\ \text { Power Amplifier / Cavity } & 250 \mathrm{~kW} & \\ \text { Energy Range, MeV } & \mathbf{4 0 0} & \mathbf{1 , 5 0 0} \\ \beta & 0.7131 & 0.9230 \\ \text { Revol. Frequency, MHz } & 0.2649 & 0.3428 \\ \text { Revolution Period, } \mu \mathrm{s} & 3.78 & 2.92 \\ \text { RF Frequency, MHz } & 6.357 & 8.228 \\ \text { Peak Beam Current, Amp } & 4.24 & 5.49 \\ \text { Peak Beam Power, MW } & 2.12 & 2.75\end{array}$

\section{Extraction from the FFAG}

It is simply required to extract the whole beam in one single turn. At the moment of extraction the revolution period is $2.92 \mu \mathrm{s}$ with a beam gap of about $300 \mathrm{~ns}$. Just prior to extraction, a 1-kG, 1.5-m long Kicker Magnet is rapidly turned on in correspondence of the beam gap. The beam is kicked outward and enters a Septum Magnet located upstream in

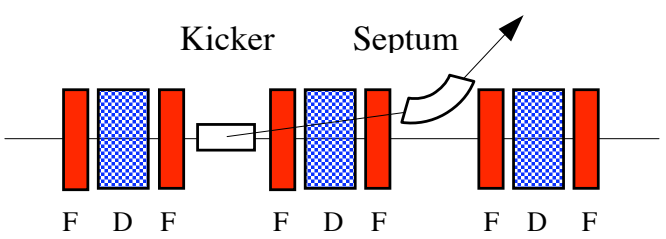
the next $2.5-\mathrm{m}$ long straight where it is initially displaced outward by about $10 \mathrm{~cm}$ (see Figure 1\%). The Septum Magnet is $1.5 \mathrm{~m}$ long and has a field of $10 \mathrm{kG}$. At the exit, the beam is displaced by $30 \mathrm{~cm}$ from the extraction orbit. This operation is repeated only one time at the repetition rate of $2.5 \mathrm{~Hz}$. Thus the Kicker Magnet has a rise-time of about 300 ns, or less. The Kicker field remains constant for the duration of the beam pulse (about $2.6 \mu \mathrm{s}$ ), and it is finally reset to zerovalue in about $100 \mathrm{~ms}$, to be fired again the next cycle.

\section{Acknowledgements}

The Author of this technical Report is grateful for the comments received from many people, and in particular wishes to acknowledge contributions from $\mathrm{N}$. Tsoupas concerning the design of the FFAG magnets, from M. Blaskiewicz for the design of the acceleration cycle, from D. Trbojevic for comments concerning the choice of the ring lattice, and from E. Courant for checking the validity of the method of adjusted field profile for the chromatic cancellation. 


\section{References}

[1] K.R. Symon et al., Phys. Rev. 103, 1837 (1956). Also, T. Ohhawa (1958). Rev. Sci. Instr. 29, 108.

[2] The MURA Staff. Proc. Intern. Conf. On High Energy Accelerators, Brookhaven, 1961. USAEC, Washington, 1961, page 57 and 344.

[3] See for instance, Proc. Of the Workshop on Accelerators for Future Spallation Neutron Sources, Vol. II B, Feb. 16-20, 1993. Picacho Plaza, Santa Fe, NM.

K. Ziegler p. 1252, and B. Kustom p. 1264.

[4] S. Machida et al., "Status of the $150 \mathrm{MeV}$ FFAG Synchrotron", PAC 2003, Portland, Oregon, May 12-16, 2003.

[5] See for instance, A. G. Ruggiero, "Design Considerations on a Proton Superconducting Linac", BNL 62312 (Informal Report), Aug. 1995. BNL Interdepartmental Study of a Spallation Neutron Source.

[6] AGS Super Neutrino Beam Facility, (NWG Report-II), Editor D. Raparia. BNL 71228 (Informal Report), April 2003.

[7] A.G. Ruggiero et al., "Design of a 1.2-GeV SCL as New Injector for the BNL AGS", BNL 71706-2003-CP. Submitted to SRF 2003 Workshop, Travemunde (Lubeck), Germany. Sept. 8-12, 2003.

[8] The SNS Project: http://www.sns.gov

[9] A.G. Ruggiero,"Review of Options for the SCL for the AGS Upgrade", BNL Internal Report, C-A/AP/\#151. April 2004.

[10] A.G. Ruggiero,"Cancellation of the Chromatic Effects with proper Field Profile", BNL Internal Report, C-A/AP/\#148. April 2004.

[11] MATHEMATICA, Wolfram Research, http://www.wolfram.com 